MATHEMATICS OF COMPUTATION

Volume 71, Number 240, Pages 1529-1543

S 0025-5718(01)01362-X

Article electronically published on November 19, 2001

\title{
AVOIDING THE ORDER REDUCTION \\ OF RUNGE-KUTTA METHODS \\ FOR LINEAR INITIAL BOUNDARY VALUE PROBLEMS
}

\author{
M. P. CALVO AND C. PALENCIA
}

\begin{abstract}
A new strategy to avoid the order reduction of Runge-Kutta methods when integrating linear, autonomous, nonhomogeneous initial boundary value problems is presented. The solution is decomposed into two parts. One of them can be computed directly in terms of the data and the other satisfies an initial value problem without any order reduction. A numerical illustration is given. This idea applies to practical problems, where spatial discretization is also required, leading to the full order both in space and time.
\end{abstract}

\section{INTRODUCTION}

Let us consider an abstract initial value problem (IVP)

$$
\left\{\begin{array}{l}
u^{\prime}(t)=A u(t)+f(t), \quad 0 \leq t \leq T, \\
u(0)=u_{0},
\end{array}\right.
$$

where $A: D(A) \subset X \rightarrow X$ is the infinitesimal generator of a $C_{0}$-semigroup in a Banach space $X, f:[0, T] \rightarrow X$ and $u_{0} \in X$. It is well known that many evolutionary problems of practical interest, either hyperbolic or parabolic, can be written in this form 28 .

Problem (1.1) is integrated in time by using a Runge-Kutta (RK) method with Butcher array

$$
\begin{array}{l|l}
\mathbf{c} & \mathcal{A} \\
\hline & \mathbf{b}^{T}
\end{array}
$$

with $\mathbf{c}=\left(c_{i}\right)_{i=1}^{s} \in \mathbb{R}^{s}, \mathbf{b}=\left(b_{i}\right)_{i=1}^{s} \in \mathbb{R}^{s}, \mathcal{A}=\left(a_{i j}\right)_{i, j=1}^{s} \in \mathbb{R}^{s \times s}$. Given an approximation $u_{n} \in X$ to $u\left(t_{n}\right), 0 \leq t_{n}<T$, the numerical approximation $u_{n+1}$ to $u\left(t_{n+1}\right)$ at $t_{n+1}=t_{n}+k_{n} \leq T$ is defined by

$$
u_{n+1}=u_{n}+k_{n}\left(\mathbf{b}^{T} \otimes A\right) U_{n}+k_{n}\left(\mathbf{b}^{T} \otimes I\right) F\left(t_{n}\right),
$$

where $F\left(t_{n}\right)=\left(f\left(t_{n}+k_{n} c_{i}\right)\right)_{i=1}^{s}$ and $U_{n} \in X^{s}$ is the solution of

$$
U_{n}=\mathbf{e} \otimes u_{n}+k_{n}(\mathcal{A} \otimes A) U_{n}+k_{n}(\mathcal{A} \otimes I) F\left(t_{n}\right),
$$

Received by the editor January 14, 2000 and, in revised form, November 30, 2000.

2000 Mathematics Subject Classification. Primary 65M12, 65M20.

Key words and phrases. Abstract initial boundary value problems, Runge-Kutta, order reduction.

This research has been supported by DGICYT under project PB95-705 and by Junta de Castilla y León under project VA36/98.

(C)2001 American Mathematical Society 
with $\mathbf{e}=[1, \ldots, 1]^{T} \in \mathbb{R}^{s}$. Here, and throughout the paper, $I$ stands for the identity operator in any space. It is known that for sufficiently small step-sizes $k_{n}$, (1.4) possesses a unique solution [5]. Let $u \in C^{p+1}([0, T], X)$ be a solution of (1.1), $p$ being the classical order of the method. Under natural stability requirements, for fixed step-sizes $k_{n}=k$ and homogeneous problems (i.e., $f=0$ ), the error $\left\|u\left(t_{n}\right)-u_{n}\right\|$ behaves like $O\left(k^{p}\right)$ [1]. However, for nonhomogeneous problems and unbounded operators $A$, it turns out that the classical order $p$ is not attained [10, 24]. 32. This is the so-called order reduction phenomenon. In fact, for nonhomogeneous problems the order of convergence is rather governed by the stage order $q$ of the method. Set

$$
\mathbf{w}_{l}=\mathbf{c}^{l}-l \mathcal{A} \mathbf{c}^{l-1}, \quad 1 \leq l \leq p,
$$

where $\mathbf{c}^{l}=\left(c_{i}^{l}\right)_{i=1}^{s}$ and $\mathbf{c}^{0}=\mathbf{e}$. Recall that $q$ is defined as the largest integer for which $\mathbf{w}_{l}=0,1 \leq l \leq q$. Typically the order of convergence is $\min \{p, q+1+\theta\}$ with $0 \leq \theta<2$ (possibly $\theta$ is not integer) 24. Let us point out that, in the context of PDEs, this order reduction does not occur in the interior of the spatial domain 21].

IVPs of the form (1.1) are a particular instance of the more general initial boundary value problems (IBVPs)

$$
\begin{cases}u^{\prime}(t)=\widetilde{A} u(t)+f(t), & 0 \leq t \leq T, \\ u(0)=u_{0}, & 0 \leq t \leq T, \\ \partial u(t)=g(t), & \end{cases}
$$

where $\widetilde{A}: D(\widetilde{A}) \subset X \rightarrow X$ is a linear extension of $A, \partial$ is a linear mapping from $D(\widetilde{A})$ to another Banach space $Y$ and $g:[0, T] \rightarrow Y$. The precise conditions to be fulfilled by these operators are described in Section 2 (see also [5]). Notice that the IVPs (1.1) arising in the applications always correspond to certain natural IBVPs (1.5) with $g=0$. When (1.5) is integrated in time with the RK method (1.2), the order of convergence is $\min \{p, q+\theta\}$ with $0 \leq \theta<2[5$. Thus, for IBVPs the order reduction is more severe than for IVPs.

Different remedies have been proposed in the literature to avoid the order reduction [1, 2, 3, 5, 12, 19, 20, 30, 32]. In the present paper a new strategy yielding the full order $p$ in the time integration of (1.5) is introduced. This strategy cannot be applied directly to the IVP (1.1). However, this is not a serious drawback since in practice, as we mentioned above, (1.1) can be written in the format (1.5).

The basic idea is as follows. The solution of (1.5) is decomposed as $u(t)=$ $v(t)+u^{*}(t)$, where $v(t)$ can be computed directly in terms of the data and $u^{*}(t)$ is the solution of a suitable IVP of the form (1.1). The key point of this construction is that $u^{*}(t) \in D\left(A^{p-q}\right)$, so that there is no order reduction in the RK approximation to $u^{*}(t)$. This strategy is studied in Section 3. Since the implementation of our strategy demands the solution of additional stationary problems at each step (see Remark 3.6 below), a high cost may result. Thus, though the strategy is interesting from a theoretical point of view, the standard implementation of an RK method with appropriate stage order could be advantageous in some instances. Numerical experiments are presented in Section 4, illustrating the theoretical results. In the final Section 5 it is shown that the remedy proposed in the present paper can also be accommodated in practical situations, where full discretizations of (1.5) are 
required. It is worth mentioning here that, as different from previous approaches [2, 3], standard spatial discretizations can be used.

\section{Abstract initial Boundary Value problems}

In this section we describe briefly the setting of [28]. Let $X, Y$ be two Banach spaces. The norms in $X$ and $Y$ and the operator norms are denoted by $\|\cdot\|$. Let $\widetilde{A}: D(\widetilde{A}) \subset X \rightarrow X, \partial: D(\widetilde{A}) \subset X \rightarrow Y$ be linear operators. We assume that

H1. The operator $(\widetilde{A}, \partial): D(\widetilde{A}) \subset X \rightarrow X \times Y$ is closed. Moreover, $\partial: D(\widetilde{A}) \subset$ $X \rightarrow Y$ is onto.

H2. Set $D(A)=\operatorname{ker}(\partial)=\{x \in D(\widetilde{A}) / \partial x=0\}$. Then the restriction $A=\left.\widetilde{A}\right|_{D(A)}: D(A) \subset X \rightarrow X$ is the infinitesimal generator of a $C_{0}$-semigroup $S(t), t \geq 0$, in $X$.

It is well known that there exist $M>0$ and $\omega \in \mathbb{R}$ such that $\|S(t)\| \leq M e^{\omega t}, t \geq 0$ (see, e.g., 31]).

In $[28$ it is proved that $\mathbf{H 1} \mathbf{- H 2}$ imply the following condition: For each $\lambda>\omega$ there exists a linear and bounded operator $K(\lambda): Y \rightarrow X$ such that, for each $y \in Y, x=K(\lambda) y \in X$ is the unique solution of the problem

$$
\left\{\begin{array}{l}
(\widetilde{A}-\lambda) x=0 \\
\partial x=y
\end{array}\right.
$$

Let us denote, for an integer $r \geq 0, \widetilde{W}_{r}=D\left(\widetilde{A}^{r}\right)$ and $W_{r}=D\left(A^{r}\right)$. It turns out that for each $\lambda>\omega$ the operator $K(\lambda) \partial: \widetilde{W}_{r} \rightarrow \widetilde{W}_{r}$ is bounded, where $\widetilde{W}_{r}$ is endowed with the norm

$$
\|x\|_{\widetilde{W}_{r}}=\|x\|+\|\widetilde{A} x\|+\cdots+\left\|\widetilde{A}^{r} x\right\|, \quad x \in \widetilde{W}_{r},
$$

which is equivalent to the graph-norm of $\widetilde{A}^{r}$.

Let $T>0$ and let $f:[0, T] \rightarrow X$ be continuous and $g:[0, T] \rightarrow Y$ a $C^{1}$-mapping. Assume that $u_{0} \in \widetilde{W}_{1}$ satisfies $\partial u_{0}=g(0)$. Then the IBVP (1.5) has got a unique solution $u:[0, T] \rightarrow X[28$.

Next we give an example to illustrate these concepts (see [4, 28, for additional examples).

Example 2.1. Let us consider the initial boundary value problem for the onedimensional heat equation

$$
\begin{cases}u_{t}(x, t)=u_{x x}(x, t)+f(x, t), & x \in[0,1], \quad 0 \leq t \leq T, \\ u(x, 0)=u_{0}(x), & x \in[0,1], \\ u(0, t)=g_{0}(t), & 0 \leq t \leq T, \\ u(1, t)=g_{1}(t), & 0 \leq t \leq T,\end{cases}
$$

where $f:[0,1] \times[0, T] \rightarrow \mathbb{R}, u_{0}:[0,1] \rightarrow \mathbb{R}, g_{0}, g_{1}:[0, T] \rightarrow \mathbb{R}$ are given functions.

Problem (2.2) can be written in the abstract form (1.5) as follows: $X=L^{p}([0,1])$, $1 \leq p<+\infty$, or $X=C_{0}([0,1])$ if $p=+\infty, Y=\mathbb{R} \times \mathbb{R}, D(\widetilde{A})=W^{2, p}([0,1])=$ $\left\{\varphi \in X / \varphi^{\prime \prime} \in X\right\}, \widetilde{A} \varphi=\varphi^{\prime \prime}$ and $\partial \varphi=[\varphi(0), \varphi(1)]^{T}, \varphi \in D(\widetilde{A})$. In this example $D(A)=W^{2, p}([0,1]) \cap W_{0}^{1, p}([0,1])=\left\{\varphi \in W^{2, p}([0,1]) / \varphi(0)=\varphi(1)=0\right\}$ and for $\xi=\left[\xi_{0}, \xi_{1}\right]^{T} \in Y, K(0) \xi$ is the mapping sending $x \in[0,1]$ to $(1-x) \xi_{0}+x \xi_{1}$. Finally, for $r \geq 1, \widetilde{W}_{r}=W^{2 r, p}([0,1])$ and $W_{r}=\left\{\varphi \in W^{2 r, p}([0,1]) / \varphi^{(2 j)}(0)=\right.$ $\left.\varphi^{(2 j)}(1)=0,0 \leq j \leq r\right\}$. 
Assume that $g \in C^{m}([0, T], Y)$ and $f^{(j)} \in C^{m-j-1}\left([0, T], \widetilde{W}_{m-j-1}\right), 0 \leq j \leq m-$ 1 , for some $m \geq 1$. Notice that in practice this is a natural requirement since these conditions only demand regularity of $f^{(j)}$ but they do not impose any restriction on the values of $f^{(j)}$ at the boundary of the spatial domain. This is opposite to the unnatural assumptions $f^{(j)}(t) \in W_{m-j-1}, 0 \leq j \leq m-1,0 \leq t \leq T$ (see example above). Under certain compatibility conditions among the data $f, g$ and $u_{0}$ (see 2]) the solution $u:[0, T] \rightarrow X$ of (1.5) belongs to $C^{m}([0, T], X) \cap C\left([0, T], \widetilde{W}_{1}\right)$.

As long as $\mathbf{H 1}$ is satisfied, we can differentiate in equation (1.5) and get that $u^{\prime}(t) \in \widetilde{W}_{1}$ and

$$
\begin{cases}u^{\prime \prime}(t)=\widetilde{A} u^{\prime}(t)+f^{\prime}(t), & 0 \leq t \leq T \\ \partial u^{\prime}(t)=g^{\prime}(t), & 0 \leq t \leq T\end{cases}
$$

Moreover, since $u^{\prime}(t)=\widetilde{A} u(t)+f(t)$ and $f(t) \in \widetilde{W}_{1}$, we have $u(t) \in \widetilde{W}_{2}$ and then

$$
u^{\prime \prime}(t)=\widetilde{A}^{2} u(t)+\widetilde{A} f(t)+f^{\prime}(t) .
$$

In fact, by induction, it is easy to prove that, for $1 \leq l \leq m$ and $0 \leq t \leq T$, $u(t) \in \widetilde{W}_{l}, u^{(l-1)}(t) \in \widetilde{W}_{1}$ and

$$
\left\{\begin{array}{l}
u^{(l)}(t)=\widetilde{A}^{l} u(t)+\sum_{j=0}^{l-1} \widetilde{A}^{l-j-1} f^{(j)}(t) \\
\partial u^{(l)}(t)=g^{(l)}(t)
\end{array}\right.
$$

This also yields

$$
\widetilde{A}^{l} u(t)=u^{(l)}(t)-\sum_{j=0}^{l-1} \widetilde{A}^{l-j-1} f^{(j)}(t)
$$

so that

$$
\partial \widetilde{A}^{l} u(t)=g^{(l)}(t)-\sum_{j=0}^{l-1} \partial \widetilde{A}^{l-j-1} f^{(j)}(t)
$$

In summary, we have shown the way to obtain the boundary values of $\widetilde{A}^{l} u$ in terms of the data $f$ and $g$ and their derivatives.

In the next section we will use the related expression for $\lambda>\omega$

$$
\partial(\lambda-\widetilde{A})^{l} u(t)=\lambda^{l} g(t)+\sum_{j=1}^{l}\left(\begin{array}{l}
l \\
j
\end{array}\right) \lambda^{l-j}(-1)^{j}\left[g^{(j)}(t)-\sum_{i=0}^{j-1} \partial \widetilde{A}^{j-i-1} f^{(i)}(t)\right],
$$

which is a direct consequence of (2.4).

\section{The MAIN RESUlT}

Let $u:[0, T] \rightarrow X$ be a solution of (1.5). We try to write $u=v+u^{*}$, where $u^{*}:[0, T] \rightarrow X$ is the solution of a suitable IVP

$$
\left\{\begin{array}{l}
u^{* \prime}(t)=A u^{*}(t)+f^{*}(t) \\
u^{*}(0)=u_{0}^{*}
\end{array}\right.
$$


and where $v:[0, T] \rightarrow X, u_{0}^{*}$ and $f^{*}:[0, T] \rightarrow X$ can be computed from the data $u_{0}, f$ and $g$ in such a way that the RK method applied to (3.1) does not suffer from order reduction.

This idea is frequently used with $v(t)=E g(t)$, where $E: Y \rightarrow X$ is a linear operator such that $\partial E y=y$ for $y \in Y$. This choice leads to the initial value problem

$$
\left\{\begin{array}{l}
u^{* \prime}(t)=A u^{*}(t)+f(t)-E g^{\prime}(t)+\widetilde{A} E g(t), \\
u^{*}(0)=u_{0}-E g(0) .
\end{array}\right.
$$

Since $u^{*} \in D(A)$, then the order of the RK discretization of (3.2) is $k^{q+1}$ (see [5, 24]). Thus, for $q<p-1$ and this choice of $v$, the order reduction still appears for $u^{*}$.

Our goal is to construct a more appropriate correcting term $v$. To this end we define the operators $C_{r}(\lambda): \widetilde{W}_{r} \rightarrow X$ as

$$
C_{r}(\lambda)=\sum_{s=0}^{r-1}(\lambda-A)^{-s} K(\lambda) \partial(\lambda-\widetilde{A})^{s}, \quad \lambda>\omega, \quad r \geq 1 .
$$

Before defining $v$ we give the following technical lemma which will be used in the proof of Theorem 1 .

Lemma 3.1. Let $r \geq 1, \lambda>\omega$. Then

(i) $C_{r}(\lambda)$ is a bounded operator from $\widetilde{W}_{r}$ to $\widetilde{W}_{r}$.

(ii) For $0 \leq j \leq r-1$ and $x \in \widetilde{W}_{r}$ it holds that

$$
\partial(\lambda-\widetilde{A})^{j} C_{r}(\lambda) x=\partial(\lambda-\widetilde{A})^{j} x .
$$

Proof. Since $K(\lambda) \partial$ is a bounded operator from $\widetilde{W}_{r-s}$ to $\widetilde{W}_{r-s}, 0 \leq s \leq r-1$, it is clear that $(\lambda-A)^{-s} K(\lambda) \partial(\lambda-\widetilde{A})^{s}$ is a bounded operator from $\widetilde{W}_{r}$ to $\widetilde{W}_{r}$. This proves (i).

Let $x \in \widetilde{W}_{1}$. Since $(\lambda-\widetilde{A}) K(\lambda) \partial x=0$ and $(\lambda-\widetilde{A})(\lambda-A)^{-1} x=x$, it turns out that, for $x \in \widetilde{W}_{r}, r \geq 2$,

$$
\begin{aligned}
(\lambda-\widetilde{A}) C_{r}(\lambda) x & =(\lambda-\widetilde{A}) K(\lambda) \partial x+\sum_{s=1}^{r-1}(\lambda-\widetilde{A})(\lambda-A)^{-s} K(\lambda) \partial(\lambda-\widetilde{A})^{s} x \\
& =C_{r-1}(\lambda)(\lambda-\widetilde{A}) x .
\end{aligned}
$$

We now proceed by induction in the proof of (ii). For $x \in \widetilde{W}_{1}$,

$$
C_{1}(\lambda) x=K(\lambda) \partial x
$$

and (ii) for $r=1$ is a direct consequence of the definition of $K(\lambda)$. Let us now assume that (ii) holds for $r-1, r \geq 2$. Let $x \in \widetilde{W}_{r}$. By (3.4), for $1 \leq j \leq r-1$

$$
\partial(\lambda-\widetilde{A})^{j} C_{r}(\lambda) x=\partial(\lambda-\widetilde{A})^{j-1} C_{r-1}(\lambda)(\lambda-\widetilde{A}) x .
$$

Then using (ii) with $r-1$, we conclude that

$$
\partial(\lambda-\widetilde{A})^{j} C_{r}(\lambda) x=\partial(\lambda-\widetilde{A})^{j-1}(\lambda-\widetilde{A}) x=\partial(\lambda-\widetilde{A})^{j} x .
$$

Moreover, (ii) is clear for $j=0$ since $\partial(\lambda-A)^{-1}=0$.

We are now in a position to define the correcting term. Assume that the solution $u$ of $(1.5)$ belongs to $C^{p+1}\left([0, T], \widetilde{W}_{r}\right)$ for some $1 \leq r \leq p-q$. We want to stress 
again that, in practice, this condition is natural, since it only demands the solution be smooth both in time and space. We define

$$
v(t)=C_{r}(\lambda) u(t), \quad 0 \leq t \leq T,
$$

where $C_{r}(\lambda)$ is given by (3.3) with $\lambda>\omega$ fixed. Observe that $r=1$ corresponds to the usual correction mentioned above with $E=K(0)$.

Notice that, due to (2.5), $v(t)$ can certainly be computed in terms of $f$ and $g$ and their derivatives. For this choice of $v$ it turns out that

$$
\begin{aligned}
f^{*}(t) & =f(t)-C_{r}(\lambda) u^{\prime}(t)+\widetilde{A} C_{r}(\lambda) u(t), \\
u_{0}^{*} & =\left[I-C_{r}(\lambda)\right] u_{0} .
\end{aligned}
$$

Assume also that $f \in C^{p}([0, T], X), g \in C^{p+1}([0, T], Y)$. Taking derivatives in equation (1.5) and using $\mathbf{H 1}$ as in Section 2 prove that $f^{(j)}(t) \in \widetilde{W}_{r-j-1}, 0 \leq j \leq$ $r-1,0 \leq t \leq T$ and then $f^{*}(t)$ can again be computed in terms of the data and their derivatives. More precisely,

$$
f^{*}(t)=\left[I-C_{r}(\lambda)\right] f(t)+(\lambda-A)^{-(r-1)} K(\lambda) \partial(\lambda-\widetilde{A})^{r} u(t),
$$

where $\partial(\lambda-\widetilde{A})^{r} u(t)$ is given by (2.5). If $\omega<0$, we can choose $\lambda=0$ and the simpler expression

$$
f^{*}(t)=\left[I-C_{r}(0)\right] f(t)-A^{-(r-1)} K(0)\left[g^{(r)}(t)-\sum_{j=0}^{r-1} \partial \widetilde{A}^{r-j-1} f^{(j)}(t)\right]
$$

results.

Recall that the stability function $r(z)$ of the RK method is defined by $r(z)=$ $1+z \mathbf{b}^{T}(I-z \mathcal{A})^{-1} \mathbf{e}$. Hereafter, we suppose that there exists $\bar{k}>0$ such that, for $0<k \leq \bar{k}$, the operator $I-\mathcal{A} \otimes k A: D(A)^{s} \subset X^{s} \rightarrow X^{s}$ is invertible (see, e.g., [5]). Therefore, for $0<k \leq \bar{k}$ the equations defining the RK approximations are solvable and the operator $r(k A)$ is well defined in $X$.

In the following theorem we assume that $\max k_{m} \leq \bar{k}$. Let us denote by $u_{n}^{*}$ the RK approximation to the solution of (3.1) at time level $t_{n}, 0 \leq n \leq N$. Thus, we adopt $u_{n}=u_{n}^{*}+v\left(t_{n}\right)$ as the numerical approximation to $u\left(t_{n}\right)$.

Theorem 3.2. Let $u \in C^{p+1}\left([0, T], \widetilde{W}_{r}\right), r=p-q$, be the solution of the IBVP (1.5). Assume also that $f \in C^{p}([0, T], X)$ and $g \in C^{p+1}([0, T], Y)$. Let $v:[0, T] \rightarrow$ $X$ be the correcting term defined in (3.5). Then

$$
\left\|u\left(t_{n}\right)-u_{n}\right\|=\left\|u^{*}\left(t_{n}\right)-u_{n}^{*}\right\| \leq C \rho_{n} \sum_{m=1}^{n} k_{m-1}^{p+1} I_{m}(u),
$$

where $C>0$ is a constant and

$$
\begin{aligned}
\rho_{n} & =\left\|\prod_{m=1}^{n} r\left(k_{m-1} A\right)\right\|, \\
I_{m}(u) & =\sup _{q+1 \leq l \leq p+1}\left\|u^{(l)}\right\|_{L^{\infty}\left(\left[t_{m-1}, t_{m}\right], \widetilde{W}_{r}\right)} .
\end{aligned}
$$

The constant $C$ depends on $T, A$ and the $R K$ method but it is independent of $u, f$ and $g$. 
Proof. By Lemma 1, $I-C_{r}(\lambda): \widetilde{W}_{r} \rightarrow \widetilde{W}_{r}$ is bounded. The norm of this operator is denoted by $L$.

Since $u^{*}(t)=\left[I-C_{r}(\lambda)\right] u(t), 0 \leq t \leq T$, then $u^{*} \in C^{p+1}\left([0, T], \widetilde{W}_{r}\right)$ and

$$
\left\|u^{*(l)}(t)\right\|_{\widetilde{W}_{r}} \leq L\left\|u^{(l)}(t)\right\|_{\widetilde{W}_{r}}, \quad 0 \leq l \leq p+1 .
$$

Moreover, again by Lemma 1 , we have

$$
\partial(\lambda-\widetilde{A})^{j} u^{(l)}(t)=\partial(\lambda-\widetilde{A})^{j} C_{r}(\lambda) u^{(l)}(t), \quad 0 \leq j \leq r-1,
$$

whence

$$
\partial(\lambda-\widetilde{A})^{j} u^{*(l)}(t)=0, \quad 0 \leq j \leq r-1,
$$

so that $u^{*(l)}(t) \in W_{r}$ for $0 \leq l \leq p+1,0 \leq t \leq T$. Thus, $u^{*} \in C^{p+1}\left([0, T], W_{r}\right)$ and

$$
\left\|A^{j} u^{*(l)}(t)\right\| \leq L\left\|u^{(l)}(t)\right\|_{\widetilde{W}_{r}}, \quad 0 \leq j \leq r, \quad 0 \leq l \leq p+1 .
$$

Now, the proof is straightforward by well-known results concerning RK methods for nonhomogeneous problems [5, 10] and by using (3.6).

Remark 3.3. It makes sense to carry out corrections with $1 \leq r<p-q$. Then the arguments used in the proof of Theorem 1 show that

$$
\left\|u\left(t_{n}\right)-u_{n}\right\| \leq C \rho_{n} \sum_{m=1}^{n} k_{m-1}^{q+r+1} I_{m}(u) .
$$

Furthermore the results in [5, 24] imply that if $r(\infty) \neq 1$ and under natural stability requirements, then for fixed step-sizes $k_{m}=k$

$$
\left\|u\left(t_{n}\right)-u_{n}\right\| \leq C \rho_{n} k^{q+r+1} \sum_{m=1}^{n} I_{m}(u) .
$$

Thus, in this situation the full order $p$ is achieved with $r=p-q-1$.

Remark 3.4. Fractional orders of convergence are also possible. Let us assume that there exists $\mu \in(0,1)$ such that $\widetilde{W}_{1} \subset W_{\mu}$, where $W_{\mu}$ is $D\left(\left(\omega^{*}-A\right)^{\mu}\right)$, for some $\omega^{*}>\omega$. Let us point out that this is the case indeed in many examples (see [4]). For instance in the example of equation (2.2), it turns out $\mu=1 /(2 p), 1 \leq p \leq+\infty$. It is easy to prove that $I-C_{r}(\lambda)$ maps $\widetilde{W}_{r+1}$ to $W_{r+\mu}$ continuously. Then, according to the results in [5], an extra order $\mu$ is present in (3.7) and (3.8).

Remark 3.5. If $A$ is the infinitesimal generator of a $C_{0}$-semigroup of contractions in a Hilbert space and the RK method is A-stable, then [16] 35]

$$
\rho_{n} \leq e^{t_{n} \max \{0, \omega\}} .
$$

For general $C_{0}$-semigroups in Banach spaces and fixed step-sizes the bound

$$
\rho_{n} \leq e^{t_{n} \max \{0, \omega\}} O(\sqrt{n})
$$

is optimal [11, 14, 15] and half an order of convergence could be lost. In special situations this loss might not occur (see [11]).

On the other hand, if $A$ is the infinitesimal generator of an analytic semigroup (see, e.g., [6, 14, 25, 26]), then

$$
\rho_{n} \leq e^{t_{n} \max \{0, \omega\}} O(1) .
$$

This bound is valid even for variable step-sizes if $|r(\infty)|<1$ [8, 26]. 
Remark 3.6. From the definition of $C_{r}(\lambda)$ it is clear that the computation of the initial condition $u_{0}^{*}$ and each evaluation of $f^{*}$ and the correcting term $v$ require the solution of $2 r-1$ stationary problems as (2.1). In these stationary problems, derivatives of $f$ and $g$ are involved which, in the evaluation of $v$ and $f^{*}$, may be replaced by appropriate approximations of order at least $p$. Notice also that the computation of $v(t)$ is only necessary at the time levels where output is required.

\section{Numerical illustration}

In this section we present two simple experiments to illustrate the results in Section 3, Let us consider the IBVP for the one-dimensional heat equation (2.2) in the example, with $p=+\infty$, where $f, u_{0}, g_{0}$ and $g_{1}$ are chosen for $u(x, t)=$ $\sin x /(1+t)$ to be its exact solution. As mentioned at the beginning of Section 3 . introducing the usual correcting term

$$
v(t)=E g(t)=g_{0}(t)+x\left[g_{1}(t)-g_{0}(t)\right],
$$

and defining $u^{*}=u-v$, the original problem is transformed into the IBVP with homogeneous boundary conditions

$$
\left\{\begin{array}{l}
u_{t}^{*}(x, t)=u_{x x}^{*}(x, t)+f(x, t)-g_{0}^{\prime}(t)-x\left[g_{1}^{\prime}(t)-g_{0}^{\prime}(t)\right], \\
u^{*}(x, 0)=u_{0}(x)-g_{0}(0)-x\left[g_{1}(0)-g_{0}(0)\right], \\
u^{*}(0, t)=0 \\
u^{*}(1, t)=0 .
\end{array}\right.
$$

$(x \in[0,1], 0 \leq t \leq T)$. For the spatial discretization of (4.1), given an integer $J \geq 2$, we define the uniform grid $x_{j}=j h, 1 \leq j \leq J$, in the interval $[0,1]$, where $h=1 /(J+1)$. The second derivative is approximated using the standard three-point finite differences.

For the time integration we use the singly diagonally implicit RK method proposed in [13] with Butcher array

$$
\begin{array}{c|ccc}
\gamma & \gamma & & \\
1 / 2 & 1 / 2-\gamma & \gamma & \\
1-\gamma & 2 \gamma & 1-4 \gamma & \gamma \\
\hline & \delta & 1-2 \delta & \delta
\end{array}
$$

where

$$
\gamma=\frac{1}{\sqrt{3}} \cos \left(\frac{\pi}{18}\right)+\frac{1}{2}, \quad \delta=\frac{1}{6(2 \gamma-1)^{2}} .
$$

This method has order $p=4$, stage order $q=1$, is $A$-stable and $|r(\infty)|<1$. According to the results included in [5, 24] and, since $u^{*} \in D(A)$ and $\mu=0$ (see Remark 2), the expected order of the error of the time integrator is three.

In our numerical experiments, to clearly observe the error due to the time integrator, we integrate the differential system

$$
\left\{\begin{array}{l}
u_{h}^{* \prime}(t)=A_{h} u_{h}^{*}(t)+\hat{f}_{h}^{*}(t), \quad 0 \leq t \leq T, \\
u_{h}^{*}(0)=\left[u^{*}\left(x_{1}, 0\right), \ldots, u^{*}\left(x_{J}, 0\right)\right]^{T},
\end{array}\right.
$$

where

$$
\hat{f}_{h}^{*}(t)=\left(P_{h} A-A_{h} P_{h}\right) u^{*}(x, t)+P_{h} f^{*}(t) .
$$


TABLE 1. Errors for $r=1$

\begin{tabular}{|l||c|c|c|c|c|}
\hline$h=k$ & $5.000 \mathrm{e}-02$ & $2.500 \mathrm{e}-02$ & $1.250 \mathrm{e}-02$ & $6.250 \mathrm{e}-03$ & $3.125 \mathrm{e}-03$ \\
\hline Error & $5.9752 \mathrm{e}-07$ & $7.0579 \mathrm{e}-08$ & $8.2533 \mathrm{e}-09$ & $9.8621 \mathrm{e}-10$ & $1.2009 \mathrm{e}-10$ \\
\hline Order & 3.08 & 3.10 & 3.10 & 3.04 & \\
\hline
\end{tabular}

TABLE 2. Errors for $r=2$

\begin{tabular}{|l||c|c|c|c|c|}
\hline$h=k$ & $5.000 \mathrm{e}-02$ & $2.500 \mathrm{e}-02$ & $1.250 \mathrm{e}-02$ & $6.250 \mathrm{e}-03$ & $3.125 \mathrm{e}-03$ \\
\hline Error & $5.5893 \mathrm{e}-08$ & $5.5342 \mathrm{e}-09$ & $4.6049 \mathrm{e}-10$ & $3.4250 \mathrm{e}-11$ & $2.3790 \mathrm{e}-12$ \\
\hline Order & 3.34 & 3.59 & 3.75 & 3.85 & \\
\hline
\end{tabular}

Here for $\varphi \in D(A), P_{h} \varphi(x, t)=\left[\varphi\left(x_{1}, t\right), \ldots, \varphi\left(x_{J}, t\right)\right]^{T}$ and $A_{h}$ is the three-point approximation to $A$. The exact solution of (4.2) is the restriction of the exact solution of (4.1) to the grid points, so that there is no spatial error.

In Table 1 the global errors when $h=k$ have been reported. We observe that when halving $k$ the global errors are approximately divided by 8 , as corresponding to the third order. This means that the order reduction is still present because the classical order of the RK method is four.

In our second experiment we transform the original IBVP (2.2) into the IVP (3.1) by introducing the correcting term with $r=2$

$$
v=C_{2}(0) u=E g+A^{-1} E\left[g^{\prime}-\partial f\right] .
$$

Now $u^{*}=u-v \in D\left(A^{2}\right)$ and, according to Remark 1, the global error of the time integrator should exhibit the full order four. In Table 2 the global errors for this second experiment are presented. The quantities in the last row approach the full order four as $k$ goes to zero, as expected.

\section{The FUlLY DisCRETE PROBLEM}

When solving IBVPs arising in PDEs using the method of lines, first it is necessary to discretize with respect to the spatial variable and then to time integrate the resulting system of ordinary differential equations. Then, in practice, we do not time integrate the IVP (3.1) but a discrete version of it. In the numerical experiments shown in Section 4 we have computed $u_{0}^{*}$ and $f^{*}$ exactly and then we have discretized with respect to the spatial variable. However, in general, it is not possible to compute (3.1) exactly because it would require the solution of certain stationary problems which, in most cases, must be solved numerically. In this section we prove that the result in Section 3 also applies to the fully discrete problem. As we mentioned in the introduction, one advantage of our approach is that standard spatial discretizations can be used.

Let us denote by $0<h<h_{0}$ the parameter of the spatial discretization and let $\left(X_{h},\|\cdot\|\right)$ be a family of normed spaces approximating $X[17,29$. The link between $X$ and $X_{h}$ is given by certain linear mappings $P_{h}: X \rightarrow X_{h}$. For $x \in X, P_{h} x$ is the approximation to $x$ in $X_{h}$. The operator $A: D(A) \rightarrow X$ is approximated by linear operators $A_{h}: X_{h} \rightarrow X_{h}$. As usual [17] 29], we assume that the semigroups $e^{t A_{h}}$ are uniformly bounded, i.e., $\left\|e^{t A_{h}}\right\| \leq M e^{\omega t}, t \geq 0$, for some $M$ and $\omega$ independent of $h$. In this situation, the consistency of the spatial discretization is measured by

$$
\varphi_{s}(h):=\left\|\left(\omega^{*}-A_{h}\right)^{-1}\left(A_{h} P_{h}-P_{h} A\right)\right\|_{W_{s} \rightarrow X_{h}},
$$

for some $s>0$ and $\omega^{*}>\omega$. Recall that $W_{s}=D\left(A^{s}\right)$. 
For instance, in the case of finite elements of degree $2 s-1$ and a second-order elliptic operator $A$, it turns out that $\varphi_{s}(h)=O\left(h^{2 s}\right)$ in the $L_{2}$-norm [36]. If a finite difference scheme of order $2 s-2$ is used instead, again for an elliptic operator $A$ of second order, we have

$$
\left\|\left(A_{h} P_{h}-P_{h} A\right)\right\|_{W_{s} \rightarrow X_{h}}=O\left(h^{2 s-2}\right)
$$

and, since $\left\|\left(\omega^{*}-A_{h}\right)^{-1}\right\| \leq M /\left(\omega^{*}-\omega\right)$, then $\varphi_{s}(h)=O\left(h^{2 s-2}\right)$.

We also assume that $\varphi_{s}(h)$ balances the behaviour of $\left\|A_{h}\right\|$ in the sense that

$$
\left\|A_{h}^{m-1}\left(A_{h} P_{h}-P_{h} A\right)\right\|_{W_{m} \rightarrow X_{h}} \leq \lambda, \quad 1 \leq m \leq s,
$$

for some $\lambda>0$ independent of $h$. This condition is clearly satisfied in the context of either finite elements or finite differences mentioned above, where $\left\|A_{h}\right\|=O\left(h^{-2}\right)$.

In the present context of IBVPs also the operators $C_{r}(\lambda)$ must be approximated. In practice, this can be done by using either boundary elements [9, 18] or finite differences [22, 23] for discretizing $K(\lambda) \partial$. Thus, we assume that $P_{h} f^{*}(t), P_{h} v(t)$ and $P_{h} u_{0}^{*}$ are approximated by $f_{h}^{*}(t), v_{h}(t)$ and $u_{h, 0}^{*}$ in $X_{h}$. For solutions $u$ taking values in $\widetilde{W}_{s}$ we denote by $\psi_{s}(h)$ the error of these approximations, i.e.,

$$
\begin{aligned}
\left\|P_{h} f^{*}(t)-f_{h}^{*}(t)\right\| & =O\left(\psi_{s}(h)\right), \\
\left\|P_{h} u_{0}^{*}-u_{h, 0}^{*}\right\| & =O\left(\psi_{s}(h)\right), \\
\left\|P_{h} v(t)-v_{h}(t)\right\| & =O\left(\psi_{s}(h)\right),
\end{aligned}
$$

as $h \rightarrow 0+$, uniformly in $0 \leq t \leq T$.

After the spatial discretization of (3.1), we have to time integrate the differential system

$$
\left\{\begin{array}{l}
u_{h}^{* \prime}(t)=A_{h} u_{h}^{*}(t)+f_{h}^{*}(t), \\
u_{h}^{*}(0)=u_{h, 0}^{*}
\end{array}\right.
$$

Let us denote by $u_{h, n}^{*}$ the RK approximation to the solution of (15.3) at time level $t_{n}=n k$. The fully discrete approximation to $u\left(t_{n}\right)$ is thus defined by $u_{h, n}=$ $u_{h, n}^{*}+v_{h}\left(t_{n}\right)$.

The fully discrete scheme is said to be stable if there exist $\bar{k}>0$ and $\rho=\rho(T)>0$ such that, for $0<k \leq \bar{k}$, the operator $I-\mathcal{A} \otimes k A_{h}: X_{h} \rightarrow X_{h}$ is invertible, $0<h<h_{0}$, and

$$
\left\|r\left(k A_{h}\right)^{n}\right\| \leq \rho, \quad 0 \leq n k \leq T .
$$

Under these assumptions we can establish the result for the full discretization.

Theorem 5.1. Let $u \in C^{p+1}\left([0, T], \widetilde{W}_{r}\right), r=p-q$, be the solution of the IBVP (1.5). Assume also that $f \in C^{p}([0, T], X), g \in C^{p+1}([0, T], Y)$. Assume that (5.2) is satisfied for $s=r$ and that the fully discrete scheme is stable. Then, for $0<\bar{k} \leq \bar{k}$,

$$
\left\|P_{h} u\left(t_{n}\right)-u_{h, n}\right\| \leq C t_{n}\left[k^{p}+\varphi_{r}(h)+\psi_{r}(h)\right],
$$

where $C=C(u, \lambda, \rho)$.

Proof. For simplicity in the proof we suppose $\omega<0$.

Set $e_{h, n}^{*}=P_{h} u^{*}\left(t_{n}\right)-u_{h, n}^{*}$. From the definition of $u_{h, n}$ and the relation $u^{*}(t)=$ $u(t)-v(t)$ it is clear that

$$
\left\|P_{h} u\left(t_{n}\right)-u_{h, n}\right\| \leq\left\|e_{h, n}^{*}\right\|+\left\|P_{h} v\left(t_{n}\right)-v_{h}\left(t_{n}\right)\right\| .
$$


The second term is bounded by $O\left(\psi_{r}(h)\right)$ and only $e_{h, n}^{*}$ must be analyzed. Let $\bar{u}_{h, n+1}^{*}$ be the RK approximation to the solution of

$$
x_{h}^{\prime}(t)=A_{h} x_{h}(t)+f_{h}^{*}(t)
$$

at $t_{n+1}$, starting from $x_{h}\left(t_{n}\right)=P_{h} u^{*}\left(t_{n}\right)$. Then,

$$
e_{h, n+1}^{*}=\left(\bar{u}_{h, n+1}^{*}-u_{h, n+1}^{*}\right)+\left(P_{h} u^{*}\left(t_{n+1}\right)-\bar{u}_{h, n+1}^{*}\right) .
$$

The first two terms on the right-hand side are numerical solutions of (5.4) after one step with initial conditions $P_{h} u^{*}\left(t_{n}\right)$ and $u_{h, n}^{*}$ respectively, so that

$$
e_{h, n+1}^{*}=r\left(k A_{h}\right) e_{h, n}^{*}+\left(P_{h} u^{*}\left(t_{n+1}\right)-\bar{u}_{h, n+1}^{*}\right) .
$$

Next, in order to use the variation-of-constants formula, it is sufficient to study the truncation term $P_{h} u^{*}\left(t_{n+1}\right)-\bar{u}_{h, n+1}^{*}$. We set $F^{*}(t)=\left(f^{*}\left(t+c_{i} k\right)\right)_{i=1}^{s}, U^{*}(t)=$ $\left(u^{*}\left(t+c_{i} k\right)\right)_{i=1}^{s}$ and $U^{* \prime}(t)=\left(u^{* \prime}\left(t+c_{i} k\right)\right)_{i=1}^{s}$ for the differential equation (3.1) and $F_{h}^{*}(t)=\left(f_{h}^{*}\left(t+c_{i} k\right)\right)_{i=1}^{s}$ for (5.4).

Direct substitution of $P_{h} u^{*}$ into the equations defining the stages of the RK approximation to the solution of (5.4) defines the defects $\tau_{h, n}$ by

$$
P_{h} U^{*}\left(t_{n}\right)=(\mathbf{e} \otimes I) P_{h} u^{*}\left(t_{n}\right)+\left(\mathcal{A} \otimes k A_{h}\right) P_{h} U^{*}\left(t_{n}\right)+k(\mathcal{A} \otimes I) F_{h}^{*}\left(t_{n}\right)+\tau_{h, n},
$$

which can also be written as

$$
\begin{aligned}
P_{h} U^{*}\left(t_{n}\right) & =P_{h}\left[(\mathbf{e} \otimes I) u^{*}\left(t_{n}\right)+k \mathcal{A} \otimes U^{* \prime}\left(t_{n}\right)\right] \\
& +k \mathcal{A} \otimes\left(A_{h} P_{h}-P_{h} A\right) U^{*}\left(t_{n}\right)+k(\mathcal{A} \otimes I)\left(F_{h}^{*}-P_{h} F^{*}\right)\left(t_{n}\right)+\tau_{h, n} .
\end{aligned}
$$

Taylor expansions of $U^{*}$ and $U^{* \prime}$ at $t_{n}$ show

$$
U^{*}\left(t_{n}\right)-(\mathbf{e} \otimes I) u^{*}\left(t_{n}\right)-k \mathcal{A} \otimes U^{* \prime}\left(t_{n}\right)=\sum_{l=q+1}^{p} \frac{k^{l}}{l !}\left(\mathbf{w}_{l} \otimes I\right) u^{*(l)}\left(t_{n}\right)+O\left(k^{p+1}\right)
$$

(recall that $\mathbf{w}_{l}=\mathbf{c}^{l}-l \mathcal{A} \mathbf{c}^{l-1}$, for $\left.q+1 \leq l \leq p\right)$. Then

$$
\begin{aligned}
\tau_{h, n} & =\sum_{l=q+1}^{p} \frac{k^{l}}{l !}\left(\mathbf{w}_{l} \otimes I\right) P_{h} u^{*(l)}\left(t_{n}\right)-k \mathcal{A} \otimes\left(A_{h} P_{h}-P_{h} A\right) U^{*}\left(t_{n}\right) \\
& -k(\mathcal{A} \otimes I)\left(F_{h}^{*}-P_{h} F^{*}\right)\left(t_{n}\right)+O\left(k^{p+1}\right) .
\end{aligned}
$$

On the other hand, the internal stages $\bar{U}_{h, n}^{*}$ for the RK approximation to the solution of (5.4) starting from $P_{h} u^{*}\left(t_{n}\right)$ satisfy

$$
\bar{U}_{h, n}^{*}=(\mathbf{e} \otimes I) P_{h} u^{*}\left(t_{n}\right)+\left(\mathcal{A} \otimes k A_{h}\right) \bar{U}_{h, n}^{*}+k(\mathcal{A} \otimes I) F_{h}^{*}\left(t_{n}\right) .
$$

Substracting (5.8) from (5.6) we get

$$
P_{h} U^{*}\left(t_{n}\right)-\bar{U}_{h, n}^{*}=\left(\mathcal{A} \otimes k A_{h}\right)\left[P_{h} U^{*}\left(t_{n}\right)-\bar{U}_{h, n}^{*}\right]+\tau_{h, n},
$$

which using (5.7) leads to

$$
\begin{aligned}
P_{h} U^{*}\left(t_{n}\right)-\bar{U}_{h, n}^{*} & =\left(I-\mathcal{A} \otimes k A_{h}\right)^{-1} \sum_{l=q+1}^{p} \frac{k^{l}}{l !}\left(\mathbf{w}_{l} \otimes I\right) P_{h} u^{*(l)}\left(t_{n}\right) \\
& -\left(I-\mathcal{A} \otimes k A_{h}\right)^{-1} k \mathcal{A} \otimes\left(A_{h} P_{h}-P_{h} A\right) U^{*}\left(t_{n}\right) \\
& -\left(I-\mathcal{A} \otimes k A_{h}\right)^{-1} k(\mathcal{A} \otimes I)\left(F_{h}^{*}-P_{h} F^{*}\right)\left(t_{n}\right)+O\left(k^{p+1}\right) .
\end{aligned}
$$


Let us now consider the equation that defines the RK approximation to the solution of (5.4) at time level $t_{n+1}$. Direct substitution of $P_{h} u^{*}$ into this equation defines the defects $\delta_{h, n+1}$ by

$$
P_{h} u^{*}\left(t_{n+1}\right)=P_{h} u^{*}\left(t_{n}\right)+k\left(\mathbf{b}^{T} \otimes A_{h}\right) P_{h} U^{*}\left(t_{n}\right)+k\left(\mathbf{b}^{T} \otimes I\right) F_{h}^{*}\left(t_{n}\right)+\delta_{h, n+1},
$$

which can be written as

$$
\begin{aligned}
P_{h} u^{*}\left(t_{n+1}\right) & =P_{h}\left[u^{*}\left(t_{n}\right)+k\left(\mathbf{b}^{T} \otimes I\right) U^{* \prime}\left(t_{n}\right)\right]+k\left(\mathbf{b}^{T} \otimes I\right)\left(A_{h} P_{h}-P_{h} A\right) U^{*}\left(t_{n}\right) \\
& +k\left(\mathbf{b}^{T} \otimes I\right)\left(F_{h}^{*}-P_{h} F^{*}\right)\left(t_{n}\right)+\delta_{h, n+1} .
\end{aligned}
$$

As $u^{*}\left(t_{n+1}\right)-u^{*}\left(t_{n}\right)-k\left(\mathbf{b}^{T} \otimes I\right) U^{* \prime}\left(t_{n}\right)=O\left(k^{p+1}\right)$, then

$$
\begin{aligned}
\delta_{h, n+1} & =-k\left(\mathbf{b}^{T} \otimes I\right)\left(A_{h} P_{h}-P_{h} A\right) U^{*}\left(t_{n}\right) \\
& -k\left(\mathbf{b}^{T} \otimes I\right)\left(F_{h}^{*}-P_{h} F^{*}\right)\left(t_{n}\right)+O\left(k^{p+1}\right) .
\end{aligned}
$$

For the numerical solution $\bar{u}_{h, n+1}^{*}$ of 5.4 starting from $P_{h} u^{*}\left(t_{n}\right)$ we have

$$
\bar{u}_{h, n+1}^{*}=P_{h} u^{*}\left(t_{n}\right)+k\left(\mathbf{b}^{T} \otimes A_{h}\right) \bar{U}_{h, n}^{*}+k\left(\mathbf{b}^{T} \otimes I\right) F_{h}^{*}\left(t_{n}\right) .
$$

Substracting (5.12) from (5.10) we get

$$
P_{h} u^{*}\left(t_{n+1}\right)-\bar{u}_{h, n+1}^{*}=\left(\mathbf{b}^{T} \otimes k A_{h}\right)\left(P_{h} U^{*}\left(t_{n}\right)-\bar{U}_{h, n}^{*}\right)+\delta_{h, n+1},
$$

which, after using (5.9) and (5.11) and some manipulations, leads to

$$
P_{h} u^{*}\left(t_{n+1}\right)-\bar{u}_{h, n+1}^{*}=K_{h}^{n}+L_{h}^{n}+M_{h}^{n}+N_{h}^{n}+O\left(k^{p+1}\right),
$$

where

$$
\begin{aligned}
K_{h}^{n} & =\left(\mathbf{b}^{T} \otimes k A_{h}\right)\left(I-k \mathcal{A} \otimes A_{h}\right)^{-1} \sum_{l=q+1}^{p} \frac{k^{l}}{l !}\left(\mathbf{w}_{l} \otimes I\right) P_{h} u^{*(l)}\left(t_{n}\right), \\
L_{h}^{n} & =-k \mathbf{b}^{T}\left(I-k \mathcal{A} \otimes A_{h}\right)^{-1}\left(F_{h}^{*}-P_{h} F^{*}\right)\left(t_{n}\right), \\
M_{h}^{n} & =-k \mathbf{b}^{T}\left(I-k \mathcal{A} \otimes A_{h}\right)^{-1}\left[I \otimes\left(A_{h} P_{h}-P_{h} A\right)\right]\left(U^{*}\left(t_{n}\right)-(\mathbf{e} \otimes I) u^{*}\left(t_{n}\right)\right),
\end{aligned}
$$

and

$$
\begin{aligned}
N_{h}^{n} & =-k \mathbf{b}^{T}\left(I-k \mathcal{A} \otimes A_{h}\right)^{-1}\left[I \otimes\left(A_{h} P_{h}-P_{h} A\right)\right](\mathbf{e} \otimes I) u^{*}\left(t_{n}\right) \\
& =\left(I-r\left(k A_{h}\right)\right) A_{h}^{-1}\left(A_{h} P_{h}-P_{h} A\right)(\mathbf{e} \otimes I) u^{*}\left(t_{n}\right) .
\end{aligned}
$$

Now the variation-of-constants formula applied to (5.5) reads

$$
e_{h, n}^{*}=r\left(k A_{h}\right)^{n}\left[P_{h} u_{0}^{*}-u_{h, 0}^{*}\right]+\sum_{j=1}^{n} r\left(k A_{h}\right)^{n-j}\left[K_{h}^{j}+L_{h}^{j}+M_{h}^{j}+N_{h}^{j}\right]+O\left(k^{p}\right) .
$$

The first term on the right-hand side of (15.14) can be bounded by $O\left(\rho \psi_{r}(h)\right)$.

The term $K_{h}^{j}$ turns out to be $O\left(k^{p+1}\right)$. To see this, notice that the equality

$$
A_{h}^{l} P_{h} u^{*(l)}=P_{h} A^{l} u^{*(l)}+\sum_{m=1}^{l} A_{h}^{m} A_{h}^{-1}\left(A_{h} P_{h}-P_{h} A\right) A^{l-m} u^{*(l)},
$$

together with (5.1) and (5.2), implies the boundedness of $\left\|A_{h}^{l} P_{h} u^{*(l)}\right\|$ for $1 \leq l \leq r$. The proof of Theorem 1 in [5] shows that $K_{h}^{j}=O\left(k^{p+1}\right)$. Then, the contribution of these terms to the error is $O\left(\rho t_{n} k^{p}\right)$. 
On the other hand, $\left\|L_{h}^{j}\right\|$ behaves as $k O\left(\psi_{r}(h)\right)$ and, since

$$
\left\|\left[I \otimes A_{h}^{-1}\left(A_{h} P_{h}-P_{h} A\right)\right]\left(U^{*}\left(t_{n}\right)-(\mathbf{e} \otimes I) u^{*}\left(t_{n}\right)\right)\right\|=k O\left(\varphi_{r}(h)\right)
$$

and the operator $\mathbf{b}^{T}\left(I-\mathcal{A} \otimes k A_{h}\right)^{-1}\left(k A_{h}\right)$ is bounded, the term $M_{h}^{j}$ behaves as $k \varphi_{r}(h)$. Therefore, these terms contribute to the error with

$$
\rho t_{n}\left(O\left(\psi_{r}(h)\right)+O\left(\varphi_{r}(h)\right)\right)
$$

It remains to analyze

$$
\sum_{j=1}^{n} r\left(k A_{h}\right)^{n-j} N_{h}^{j}
$$

which, after summation by parts, can be written as

$$
\begin{aligned}
& \sum_{j=1}^{n-1} r\left(k A_{h}\right)^{n-j} A_{h}^{-1}\left(A_{h} P_{h}-P_{h} A\right)(\mathbf{e} \otimes I)\left(u^{*}\left(t_{j}\right)-u^{*}\left(t_{j+1}\right)\right) \\
& +A_{h}^{-1}\left(A_{h} P_{h}-P_{h} A\right) u^{*}\left(t_{n}\right)-r\left(k A_{h}\right)^{n} A_{h}^{-1}\left(A_{h} P_{h}-P_{h} A\right) u^{*}\left(t_{1}\right) .
\end{aligned}
$$

As long as (5.1) is satisfied and $\left\|u^{*}\left(t_{j}\right)-u^{*}\left(t_{j+1}\right)\right\|=O(k)$, then the norm of (5.15) is $\rho t_{n} O\left(\varphi_{r}(h)\right)$.

Remark 5.2. When using finite elements in the context of the maximum norm, it turns out that the norms in (15.2) behave like $\lambda(h)=O\left(|\ln h|^{\bar{s}}\right)$, where $\bar{s}=s+1$, for piecewise linear elements, or $\bar{s}=s$, for higher order elements [33]. This is not a serious problem since $\lambda=\lambda(h)$ enters as a factor in the error constant $C$ in Theorem 5.1.

Remark 5.3. For simplicity we have assumed that the fully discrete scheme is stable. In practice, $\rho$ might depend on $h$ and $k$. This dependence has been widely studied in the literature (see references in Remark 3.5 and, for second-order elliptic operators and the maximum norm, 7] for finite differences and [27, 34 36 for finite elements). Typically $\rho$ exhibits at most weak singularities when $h$ and $k$ go to 0 . Thus, as $\rho$ enters again as a factor in $C$, the full order of convergence could be slightly spoiled, due to stability reasons.

Remark 5.4. If $r(\infty) \neq 1$, then the optimal order $p$ may be achieved with $r=$ $p-q-1$ (see Remark 3.3).

Remark 5.5. Fixed step-sizes are required for the treatment of (5.15), when summation by parts applies. However, in cases for which

$$
\left\|\left(A_{h} P_{h}-P_{h} A\right) u^{*}(t)\right\|=O\left(\varphi_{r}(h)\right)
$$

(for instance when finite differences are used), the joint contribution $M_{h}^{n}+N_{h}^{n}$ can be analyzed without using summation by parts. In these situations variable step-sizes are also allowed.

Remark 5.6. If $\widetilde{W}_{1} \subset W_{\mu}, 0<\mu<1$, and $r<p-q$, then a fractional order in time occurs as in Remark 3.4 In fact, by interpolating the two inequalities

$$
\begin{gathered}
\left\|A_{h} P_{h} \xi\right\| \leq \lambda\|\xi\|+\left\|P_{h}\right\|\|A \xi\|, \\
\left\|P_{h} \xi\right\| \leq\left\|P_{h}\right\|\|\xi\|
\end{gathered}
$$


$\xi \in W_{1}$, we deduce that when $\left\|P_{h}\right\|=O(1)$ (a standard situation),

$$
\left\|\left(\omega-A_{h}\right)^{\mu^{\prime}} P_{h} \xi\right\| \leq C_{\mu^{\prime}}\|\xi\|_{\mu},
$$

$\xi \in W_{\mu}$, for all $\mu^{\prime}<\mu$. Thus, an extra order $\mu^{\prime}$ is present for all $\mu^{\prime}<\mu$. This explains why the extra order $\mu$ is observed in full discretizations of (3.2) (see, e.g., the numerical experiments in [24]).

\section{REFERENCES}

1. S. Abarbanel, D. Gottlieb \& M. H. Carpenter, On the removal of boundary errors caused by Runge-Kutta integration of nonlinear partial differential equations, SIAM J. Sci. Comput. 17 (1996) 777-782. MR 96m:65071

2. I. Alonso-Mallo, Rational methods with optimal order of convergence for partial differential equations, Appl. Numer. Math. 35 (2000) 265-292. CMP 2001:05

3. I. Alonso-Mallo, Explicit single step methods with optimal order of convergence for partial differential equations, Appl. Numer. Math. 31 (1999) 117-131. MR 2000d:65186

4. I. Alonso-Mallo \& C. Palencia, On the convolution operators arising in the study of abstract initial boundary value problems, Proc. Royal Soc. Edinburgh 126A (1996) 515-539. MR 97d:34068

5. I. Alonso-Mallo \& C. Palencia, Optimal orders of convergence in Runge-Kutta methods for linear, non-homogeneous PDEs with singular source terms, preprint.

6. N. Yu. Bakaev, On the stability of some general discretization methods, Dokl. Akad. Nauk. SSSR 309 (1989) 11-15; English transl. in Soviet Math. Dokl. 40 (1990). MR 91e:65104

7. N. Yu. Bakaev, Estimates for the resolvent of a multi-dimensional elliptic difference operator, Function.-Diff. Eqs., Perm' PPI (1991) 118-126. MR 94e:39001

8. N. Yu. Bakaev, On variable stepsize Runge-Kutta approximations of a Cauchy problem for the evolution equation, BIT 38 (1998) 462-485. MR 99i:65069

9. C. A. Brebbia, J. C. F. Telles \& L. C. Wrobel, Boundary element techniques. Theory and applications in engineering, Springer, Berlin, 1984. MR 89i:65002

10. P. Brenner, M. Crouzeix \& V. Thomée, Single step methods for inhomogeneous linear differential equations in Banach space, R.A.I.R.O. Anal. Numer. 16 (1982) 5-26. MR 83d:65268

11. P. Brenner \& V. Thomée, On rational approximations of semigroups, SIAM J. Numer. Anal. 16 (1979) 683-694. MR 80j:47052

12. M. H. Carpenter, D. Gottlieb, S. Abarbanel \& W. S. Don, The theoretical accuracy of RungeKutta time discretizations for the initial boundary value problem: a study of the boundary error, SIAM J. Sci. Comput. 16 (1995) 1241-1252. MR 96h:65088

13. M. Crouzeix \& P. A. Raviart, Méthodes de Runge-Kutta, Unpublished Lecture Notes, Université de Rennes (1980).

14. M. Crouzeix, S. Larsson, S. Piskarev \& V. Thomée, The stability of rational approximations of holomorphic semigroups, BIT 33 (1993) 74-84. MR 96f:65069

15. J. L. M. van Dorsselaer, J. F. B. M. Kraaijenvanger \& M. N. Spijker, Linear stability analysis in the numerical solution of initial value problems, in Acta Numer. 1993, 199-237. MR 94e:65051

16. E. Hairer \& G. Wanner, Solving ordinary differential equations II, Stiff and differentialalgebraic problems, 2nd Ed., Springer, Berlin, 1996. MR 97m:65007

17. K. Ito \& F. Kappel, The Trotter-Kato theorem and approximations of PDEs, Math. Comp. 67 (1998) 21-44. MR 98e:47060

18. C. Johnson, Numerical solution of partial differential equations by the finite element method, Cambridge University Press, Cambridge, 1987. MR 89b:65003a

19. S. L. Keeling, Galerkin/Runge-Kutta discretizations for parabolic equations with timedependent coefficients, Math. Comp. 52 (1989) 561-586. MR 90a:65239

20. S. L. Keeling, Galerkin/Runge-Kutta discretizations for semilinear parabolic equations, SIAM J. Numer. Anal. 27 (1990) 394-418. MR 91d:65140

21. Ch. Lubich \& A. Ostermann, Interior estimates for time discretizations of parabolic equations, Appl. Numer. Math. 18 (1995) 241-251. MR 96f:65124

22. A. R. Mitchell \& D. F. Griffiths, The finite difference method in partial differential equations, Wiley-Interscience, New York, 1980. MR 82a:65002 
23. K. W. Morton \& D. F. Mayers, Numerical solution of partial differential equations, Cambridge University Press, Cambridge, 1994. MR 96b:65001

24. A. Ostermann \& M. Roche, Runge-Kutta methods for partial differential equations and fractional orders of convergence, Math. Comp. 59 (1992) 403-420. MR 93a:65125

25. C. Palencia, A stability result for sectorial operators in Banach spaces, SIAM J. Numer. Anal. 30 (1993) 1373-1384. MR 94j:65109

26. C. Palencia, On the stability of variable stepsize rational approximations of holomorphic semigroups, Math. Comput. 62 (1994) 93-103. MR 94c:47066

27. C. Palencia, Maximum-norm analysis of completely discrete finite element methods for parabolic problems, SIAM J. Numer. Anal. 33 (1996) 1654-1668. MR 97e:65099

28. C. Palencia \& I. Alonso-Mallo, Abstract initial boundary value problems, Proc. Royal Soc. Edinburgh 124A (1994) 879-908. MR 95k:35090

29. C. Palencia \& J. M. Sanz-Serna, An extension of the Lax-Richtmyer theory, Numer. Math. 44 (1984) 279-283. MR 86c:65096

30. D. Pathria, The correct formulation of intermediate boundary conditions for Runge-Kutta time integration of initial boundary value problems, SIAM J. Sci. Comput. 18 (1997) 12551266. MR 98d:65100

31. A. Pazy, Semigroups of linear operators and applications to partial differential equations, Springer, Berlin, 1983. MR 85g:47061

32. J. M. Sanz-Serna, J. G. Verwer \& W. H. Hundsdorfer, Convergence and order reduction of Runge-Kutta schemes applied to evolutionary problems in partial differential equations, Numer. Math. 50 (1986) 405-418. MR 88f:65146

33. A. H. Schatz \& L. B. Wahlbin, On the quasi-optimality in $L_{\infty}$ of the $H_{1}$-projection into finite element spaces, Math. Comp. 38 (1982) 1-22. MR 82m:65106

34. A. H. Schatz, V. Thomée \& L. B. Wahlbin, Stability, analyticity, and almost best approximation in maximum norm for parabolic finite element equations, Comm. Pure Appl. Math. 51 (1998) 1349-1385. MR 99h:65171

35. B. Sz.-Nagy \& C. Foias, Harmonic Analysis of Operators on Hilbert Spaces, North-Holland, Amsterdam, 1970. MR 43:947

36. V. Thomée, Galerkin finite element methods for parabolic problems, Springer, Berlin, 1997. MR 98m:65007

Departamento de Matemática Aplicada y Computación, Universidad de Valladolid, VALLADOlid, Spain

E-mail address: maripaz@mac.cie.uva.es

Departamento de Matemática Aplicada y Computación, Universidad de Valladolid, VALLADOLID, SPAin

E-mail address: palencia@mac.cie.uva.es 